\title{
ON THE NUMBER OF SUBGROUPS OF GIVEN ORDER IN A FINITE $p$-GROUP OF EXPONENT $p$
}

\author{
YA. G. BERKOVICH
}

(Communicated by Warren J. Wong)

\begin{abstract}
A. Kulakoff [1] showed that a noncyclic $p$-group of order $p^{m}, p>$ 2 , contains $1+p+k p^{2}$ subgroups of order $p^{n}, 0<n<m$, where $k$ is a nonnegative integer. In this note we show that for $1<n<m-1$ a $p$-group of order $p^{m}$ and exponent $p$ contains $1+p+2 p^{2}+k p^{3}$ subgroups of order $p^{n}$.
\end{abstract}

1.

In this section $G$ denotes a group of order $p^{m}$ and exponent $p$. Let $m, n$ be natural numbers. Let $\phi(G)$ denote the Frattini subgroup of $G$ and $p^{d(G)}=$ $p^{d}=|G: \phi(G)|$. In our situation $\phi(G)=G^{\prime}=[G, G]=K_{2}(G)$. Let $K_{3}(G)=$ $\left[K_{2}(G), G\right]$. For $i \in[1, d]$ let $M_{i}=\left\{H<G|\phi(G) \leq H| G:, H \mid=p^{i}\right\}$. Obviously $M_{d}=\{\phi(G)\}$. If $\mathfrak{M}$ is a certain set of subgroups of $G$ and $F \leq G$, then $n(F)$ denotes the number of elements of $\mathfrak{M}$ which are subgroups of $F$. Obviously $n(G)=|\mathfrak{M}|$. We use the following enumeration principle of Philip Hall:

$$
n(G)=\sum_{i=1}^{d}(-1)^{i-1} p^{i(i-1) / 2} \sum_{H \in M_{i}} n(H),
$$

This identity holds for arbitrary finite $p$-groups.

In this note we prove the following.

Theorem. Let $G$ be a p-group of order $p^{m}$ and exponent $p, 2 \leq n \leq m-2$. Then the number of subgroups of order $p^{n}$ in $G$ is congruent to $1+p+2 p^{2}$ modulo $p^{3}$.

We use the following

Lemma 1. Let $G$ be a p-group of order $p^{m}$ and exponent $p, d=d(G)$.

(a) If $G$ is minimal non-Abelian then $m=3$.

(b) If $d=2$ then $\left|G: K_{3}(G)\right|=p^{3}$.

Received by the editors May 31, 1989 and, in revised form, October 12, 1989.

1980 Mathematics Subject Classification (1985 Revision). Primary 20D10; Secondary 20D60. 
(c) Let $H$ be a two-generator maximal subgroup of $G$. Then the number of two-generator maximal subgroups in $G$ and $G / K_{3}(H)$ is the same.

(d) If $\phi(G)$ is non-Abelian then it is not generated by two elements.

(e) If $d>2$ and $G$ contains a two-generator maximal subgroup $H$ then it contains exactly $p^{2}$ such subgroups.

(f) If $d=2$ and $G$ contains a two-generator maximal subgroup then $G$ contains exactly $p$ such subgroups.

Proof. (a) Since $G$ is non-Abelian it contains two noncommuting elements $x$, $y$ and $G=\langle x, y\rangle$. Since $\phi(G)=G^{\prime}=\langle[x, y]\rangle$ and $d=2$ then $m=3$.

(b) Obviously $G / K_{3}(G)$ is minimal non-Abelian; so $\left|G / K_{3}(G)\right|=p^{3}$ by (a).

(c) Let $D=K_{3}(H)$. Then $|H / D|=p^{3}$ by (b). Let $F / D$ be a non-Abelian subgroup of index $p$ in $G / D$. Suppose that $d(F)>2$. Then $|F: \phi(F)| \geq p^{3}$. Obviously $F \neq H$. So $|G: F \cap H|=p^{2}$. Between $H \cap F$ and $\phi(F)$ there is a $G$-admissible subgroup $F_{1}$ of index $p^{2}$ in $H \cap F$. Since $\left|H / F_{1}\right|=p^{3}$ then $F_{1}=D$. Since $F / F_{1}$ is Abelian and $F / D$ is non-Abelian we obtain a contradiction. Hence $d(F)=2$. Since $D<\phi(H) \leq \phi(G)$ our assertion is proved.

(d) Suppose that $d(\phi(G))=2$. Without loss we suppose that $K_{3}(\phi(G))=1$. Then $|\phi(G)|=p^{3}$ by (b). Let $L$ be a $G$-admissible subgroup of order $p^{2}$ in $\phi(G)$. Then $\left|G: C_{G}(L)\right|=p$ and $\phi(G)<C_{G}(L), \phi(G)$ is Abelian, a contradiction.

(e) In virtue of (c) we set $K_{3}(H)=1$. Then $|G|=p^{4}$ by (b) so $G$ is not extra-special. Hence $|Z(G)|=p^{2}$. Let $x \in Z(G)-\phi(G)$. Then $G=\langle x\rangle \times F$ with $F<G$ and our assertion is obvious.

(f) As in (e) we assume that $K_{3}(H)=1$ where $H$ is a two-generator maximal subgroup of $G$. Then $m=4$ and $\left|M_{1}\right|=1+p$. Since $|Z(G)|=p$ then $M_{1}$ contains only one Abelian subgroup of index $p$, and (f) is proved.

Corollary 1. Let $G$ be a p-group of order $p^{m}$ and exponent $p, 3 \leq n<m$. Then $G$ contains $k p$ two-generator subgroups of order $p^{n}$. Here $k$ is nonnegative integer.

Proof. From Lemma $1(\mathrm{e}, \mathrm{f})$ this assertion is true for $n=m-1$. If $n<m-1$ the result follows by induction using (1).

Remark. Let $G$ be as in Corollary $1,3 \leq n \leq m-2$. If every two-generator subgroup of order $p^{s}, n<s \leq m$, contains a two-generator subgroup of index $p$ then the number of two-generator subgroups of order $p^{n}$ in $G$ is divisible by $p^{2}$. For the proof it is necessary to use Lemma $1(\mathrm{~d})$.

Proof of the theorem. Suppose that the theorem is proved for all proper subgroups of $G$. Let $\mathfrak{M}$ be the set of all subgroups of order $p^{n}$ in $G$. In virtue of (1) we have

$$
n(G) \equiv \sum_{H \in M_{1}} n(H)-p \sum_{H \in M_{2}} n(H) \quad\left(\bmod p^{3}\right)
$$


(a) $n=m-2$.

(1a) $d=2$.

If $G$ contains a two-generator maximal subgroup then it contains exactly $p$ such subgroups (Lemma $1(\mathrm{f})$ ). Hence

$$
n(G) \equiv p(1+p)+1\left(1+p+p^{2}\right)-p \equiv 1+p+2 p^{2} \quad\left(\bmod p^{3}\right) .
$$

(2a) $d>2$.

If $G$ contains a two-generator maximal subgroup then by (2) and Lemma $1(\mathrm{e})$ we have

$$
n(G) \equiv p^{2}(1+p)+(1+p)\left(1+p+p^{2}\right)-p(1+p) \equiv 1+p+2 p^{2} \quad\left(\bmod p^{3}\right) .
$$

If $G$ does not contain a two-generator maximal subgroup then

$$
n(G) \equiv\left(1+p+p^{2}\right)\left(1+p+p^{2}\right)-p(1+p) \equiv 1+p+2 p^{2} \quad\left(\bmod p^{3}\right) .
$$

(b) $n<m-2$. If $d=2$, then by (2), Kulakoff's theorem and induction we have

$$
n(G) \equiv(1+p)\left(1+p+2 p^{2}\right)-p(1+p) \equiv 1+p+2 p^{2} \quad\left(\bmod p^{3}\right) .
$$

If $d>2$ then $n(G) \equiv\left(1+p+p^{2}\right)\left(1+p+2 p^{2}\right)-p(1+p)(1+p) \equiv 1+p+$ $2 p^{2}\left(\bmod p^{3}\right)$. The theorem is proved.

Probably if $2<n<m-2$ and $d>4$ then we have

$$
n(G) \equiv 1+p+2 p^{2}+2 p^{3} \quad\left(\bmod p^{4}\right)
$$

in the theorem.

2.

We apply some of the results of the previous section to irregular $p$-groups.

Lemma 2. Let $H$ be a subgroup of order $p^{n}$ and exponent $p$ in a p-group $G$, $n<p$. If $\Omega_{1}(G)=\left\langle x \in G \mid x^{p}=1\right\rangle>H$ then $H<F \leq G$ where $|F|=p^{n+1}$ and $\exp F=p$.

Proof. Let $N=N_{G}(H)$. If $x$ is an element of order $p$ in $N-H$ then $\langle x, H\rangle$ has order $p^{n+1} \leq p^{p}$ so it is regular and $\exp \langle x, H\rangle=p$. In this case we set $F=\langle x, H\rangle$. If $H=\Omega_{1}(N)$ then $H$ is characteristic in $N$ so $N=G$, a contradiction, since $\Omega_{1}(N)=H<\Omega_{1}(G)=\Omega_{1}(N)$.

Corollary 2. Let $G$ be an irregular p-group, $2<n<p-\varepsilon$ where

$$
\varepsilon= \begin{cases}1, & G \text { is of maximal class }, \\ 0, & \text { in the opposite case. }\end{cases}
$$

Then the number of two-generator subgroups of order $p^{n}$ and exponent $p$ in $G$ is divisible by $p$.

Proof. Let $\mathfrak{M}=\left\{B_{1}, \ldots, B_{t}\right\}$ be the set of all two-generator subgroups of order $p^{n}$ and exponent $p$ in $G$. Let $\mathfrak{N}=\left\{A_{1}, \ldots, A_{r}\right\}$ be the set of all subgroups of 
order $p^{n+1}$ and exponent $p$ in $G$ such that every $A_{i}$ contains a two-generator subgroup of index $p$. In virtue of Lemma 2 if the set $\mathfrak{M}$ is nonempty then $\mathfrak{N}$ is nonempty also. Let $a_{i}$ be the number of elements of $\mathfrak{M}$ contained in $A_{i}$, $i \in[1, r]$, and $b_{j}$ be the number of elements of $\mathfrak{N}$ which contain $B_{j}$. Then

$$
a_{1}+\cdots+a_{r}=b_{1}+\cdots+b_{t} \text {. }
$$

By Corollary 1 we have $a_{i} \equiv 0(\bmod p)$ for all $i \in[1, r]$. Let $N=N_{G}\left(B_{j}\right)$. Obviously all elements of $\mathfrak{N}$ containing $B_{j}$ are subgroups of $N$. We prove that $b_{j} \equiv 1(\bmod p)$. We may assume without loss that $N=G$. If $X$ is an element of order $p$ in $G-B_{j}$ then $\left\langle x, B_{j}\right\rangle$ has order $p^{n+1}$ and exponent $p$, and, obviously, $\left\langle x, B_{j}\right\rangle$ contains

$$
\left(1+p+\cdots+p^{n}\right)-\left(1+p+\cdots+p^{n-1}\right)=p^{n}
$$

subgroups of order $p$ which lie outside $B_{j}$. By [2] $G$ contains $1+p+\cdots+p^{n}+$ $k p^{n+1}$ subgroups of order $p$, where $k \geq 0$ is an integer. Hence $G$ contains $p^{n}+k p^{n+1}$ subgroups of order $p$ which are not subgroups of $B_{j}$. So

$$
b_{j}=\left(p^{n}+k p^{n+1}\right) / p^{n}=1+k p \equiv 1 \quad(\bmod p) .
$$

Hence $t \equiv b_{1}+\cdots+b_{t}=a_{1}+\cdots+a_{r} \equiv 0(\bmod p)$, and our assertion is proved.

Lemma $1(a)$ has the following.

Corollary 3. Let $G$ be a finite p-group, $\mathfrak{M}=\left\{H \leq G|| H \mid \leq p^{4}\right.$, $\left.\exp H \leq p^{2}\right\}$. If all elements of $\mathfrak{M}$ are Abelian then $\Omega_{1}(G)$ is Abelian and $C_{G}\left(\Omega_{1}(G)\right) \geq \Omega_{2}(G)$. Proof. We note that all subgroups of exponent $p$ in $G$ are Abelian by Lemma $1(a)$.

Let $H$ be a normal subgroup of exponent $p$ of maximal order in $G$. Suppose that $\Omega_{1}(G)-H$ contains an element $x$ of order $p$. Then $L=\langle x, H\rangle$ is nonAbelian of exponent $p^{2}$ by choice of $H$. Let $A$ be a minimal non-Abelian subgroup of $L$. Then $H A=L$ and $H \cap A$ is elementary of index $p$ in $A$. Since $\left|A^{\prime}\right|=p$ and $A / A^{\prime}$ is two-generator (see proof of Lemma 1(a)) then $|A \cap H| \leq p^{3},|A| \leq p^{4}, \exp A \leq p^{2} \Rightarrow A \in \mathfrak{M}$, a contradiction. Hence $H=\Omega_{1}(G)$ is elementary Abelian.

Let $y$ be an element of order $p^{2}$ in $G$. Then $M=\langle y, H\rangle$ has exponent $p^{2},|M: H|=p$, since $y^{p} \in H$. As in the preceding paragraph we obtain a contradiction. Hence $M$ is Abelian and $\Omega_{2}(G) \leq C_{G}(H)$, and the corollary is proved.

Lemma 3. Let $G$ be a finite p-group of order $p^{m}, m \geq p+2$.

(a) [3]. If $G$ contains a maximal subgroup $H$ which is absolutely regular (i.e. $\left.\left|H:\left\langle x^{p} \mid x \in H\right\rangle\right|<p^{p}\right)$, and if $G$ is not absolutely regular or of maximal class then $G=H \Omega_{1}(G)$.

(b) $[2,4]$. Suppose $p+1<n<m, G$ is not of maximal class. Then the number of subgroups of maximal class and order $p^{n}$ in $G$ divisible by $p^{2}$. 
(c) If $G$ is of maximal class then the number of subgroups of order $p^{p-1}$ and exponent $p$ in $G$ is equal to $1+k p^{2}$, where $k$ is a nonnegative integer. In this case $G$ does not contain a normal subgroup of order $p^{p}$ and exponent $p$.

(d) If $G$ does not contain a normal subgroup of order $p^{p}$ and exponent $p$ then $G$ is absolutely regular or of maximal class.
Assertions
(c) and
(d) are proved in [3].

Corollary 4. Suppose that the irregular p-group $G$ is not of maximal class, $|G|=$ $p^{m}, m>p+2$. If $\mathfrak{M}=\left\{H<G|| H \mid=p^{p-1}, \exp H=p\right\}$ then $|\mathfrak{M}| \equiv 1+p$ $\left(\bmod p^{2}\right)$.

Proof. By Lemma 3(d) the group $G$ contains a normal subgroup $L$ of order $p^{p}$ and exponent $p$. If $L=\Omega_{1}(G)$ then our assertion follows from Kulakoff's theorem (which is true for noncyclic groups of exponent 2 also). In virtue of Lemma 3(a) $G$ has no absolutely regular maximal subgroup. Suppose the corollary is true for all proper subgroups of $G$. By (1) we obtain

$$
n(G) \equiv \sum_{H \in M_{1}} n(H)-p \quad\left(\bmod p^{2}\right)
$$

If $M_{1}$ does not contain an element of maximal class then by induction and (4) we have

$$
n(G) \equiv(1+p)(1+p)-p \equiv 1+p \quad\left(\bmod p^{2}\right) .
$$

If $M_{1}$ contains an element of maximal class then it contains exactly $p^{2}$ such elements (Lemma 3(b)) and for any such subgroup $F$ we have $n(F) \equiv 1$ $\left(\bmod p^{2}\right)$. Hence by $(4)$ and induction we have

$$
n(G) \equiv p^{2}+(1+p)(1+p)-p \equiv 1+p \quad\left(\bmod p^{2}\right),
$$

and the corollary is proved.

\section{ACKNOWLEDGMENT}

My deep thanks to the referee for many corrections and useful comments.

\section{REFERENCES}

1. A. Kulakoff, Über die Anzahl der eigentlichen Untergruppen und der Elemente von gegebener Ordnung in p-Gruppen, Math. Ann. 104 (1931), 778-793.

2. Ya. G. Berkovich, A generalization of the theorems of Hall and Blackburn and their applications to non-regular p-groups, Izv. Akad. Nauk SSSR Ser. Mat. 35 (1971), 800-831. (Russian)

3. N. Blackburn, Generalizations of certain elementary theorems on p-groups, Proc. London Math. Soc. 11 (1961), 1-22.

4. N. Blackburn, Note on a paper of Berkovich, J. Algebra 24 (1973), 323-334.

Rostov-ON-Don, 344006, PR. ENGELS 111/18, USSR 